better understand the service developments and participant's aspirations for day services.

It was found that introducing five additional patient services Blood transfusion clinic; Progressive Supranuclear Palsy support group; Motor Neurone Disease clinic; Multi System Atrophy support group and a Carers' support group - led to a $27 \%$ increase in attendances. Staff, patients and their families were recruited to three research projects by three hospice staff as a core part of their role, and five staff contributed to qualitative studies or data collection. The qualitative data indicated that staff were pioneering the new well-coordinated services but were challenged by the time available to capture data. The management team had a vision for further expansion of sustainable services and innovative approaches of working in partnership with the local acute trust and community care providers.

In conclusion, a diversification of day services offered to patients and carers can be facilitated with engaged managerial and professional staff without a significant increase in core workforce.

\section{P-195 PALLIATIVE CARE AS AN INTRINSIC PART OF INTEGRATED CARE - DOES THE MODEL FIT?}

Carolanne Brannan, Nigel Dodds. St Joseph's Hospice, London, UK

\subsection{6/bmjspcare-2016-001245.217}

Introduction Integrated Care(IC) (Ham and Alderwick, 2015) is a model of delivering health and social care, adopted by a number of Clinical Commissioning Groups across East London. This facilitates professionals to work with their external partners, to streamline the patient pathway, and improve patient outcomes. Within this model it is expected that multi-professional teams within the acute and community services, spanning all disciplines, work in collaboration by meeting regularly to proactively plan holistic, person-centred care, rather than focus on separate diseases or co-morbidities, or psycho-social issues, thus aiming to avoid working in silos, in relation to patient care.

Aims One of the main aims of IC is to work towards agreed strategies to avoid hospital admission, and support patients to remain in their own homes. St Joseph's Hospice community palliative care team are active partners in the integrated care teams, and work with other health and social care providers to ensure patients in the last years of life are enabled to be cared for, and die in their preferred place.

Methods In this presentation we will describe how all relevant professional groups, from all care settings, and involved in patient care meet monthly within every GP practice across the borough in Integrated Care meetings. The focus of these meetings is patient focused, where the most vulnerable patients within the practice are discussed, and a holistic plan of care is agreed using the knowledge and expertise of all the professionals present. This model has replaced palliative care specific meetings where the meeting was focused only on patients approaching the end of life. Through these changes, we will demonstrate the changing nature of referrals to the community palliative care team, which may have implications for the future direction of the hospice.

\section{P-196 CLINIC OR HOME}

Linda Gregory, Debbie Bolton. St Catherine's Hospice, Preston, UK

\subsection{6/bmjspcare-2016-001245.218}

Background In September 2012 the increasing number of patients referred to St Catherine's Hospice Clinical Nurse Specialist (CNS) service, gave the opportunity to explore another option for patients to receive an assessment other than at home. An outpatient clinic service was commenced.

Aims To enable the CNS team to be more responsive to patient referrals. Assessing patients who are well enough in clinic will allow more time for those patients who need to be seen in their own home. A range of other benefits and also some challenges were identified:

- Maintain patient independence

- To optimise patient choice

- It supports the first introduction to a hospice

- Reduce travel costs for CNS team

- Patient may not have transport

- The patient may not feel emotionally able to attend a hospice

- Patients have so many appointments it may be more comfortable for them to be seen at home

- Precedents set by other health professionals that the CNS visits all patients at home

- A change of culture for community nurses who are accustomed to seeing patients at home.

Methods Initially the clinic idea was quite simple in its design; a weekly clinic at the hospice providing four appointment slots.

Results There have been regular audits since 2012 which resulted in further development of the CNS clinics; leading to the current service of two clinics per week. One held at the hospice and a second clinic at the premises of a local cancer charity; providing ten appointment slots per week.

Conclusion It is recognised that patients assessed in clinic are a small percentage of the CNS clinical workload. The aim is now for each CNS to have their own caseload clinic in the community. This is currently being piloted by one CNS having a fortnightly clinic in a GP practice (February 2016).

\section{P-197 THE IMPLEMENTATION OF OUTPATIENT NURSE LED CLINICS BY THE COMMUNITY HOSPICE NURSE SPECIALIST TEAM}

Paula Taylor. Wigan and Leigh Hospice, Wigan, UK

\subsection{6/bmjspcare-2016-001245.219}

During a Strategic Away Day in November 2013, the Hospice Nurse Specialist Team (HNST) at Wigan and Leigh Hospice identified the necessity to increase responsiveness to patients' needs during periods of high demand upon the service, by considering ways of increasing overall efficiency. It was agreed to introduce nurse-led clinics for those patients functionally able to attend the hospice, using the WHO performance status tool for guidance.

The HNST have provided face to face assessments in the homes of their patients for many years. Nurse-led clinics was a significant change in approach to the model of care and 
challenged the community's expectations that the community service will only be delivered in patients' homes.

The HNST comprises of three clusters, each having two nurse specialists and one associate nurse specialist and a team manager. Initially each cluster was allocated one afternoon clinic session with $4 \times 45$ minute slots per session. The use of the clinic slots was audited and recommendations made.

In the following months there was increase in the flexibility of clinic allocations and an increase in the availability of clinic rooms. There was a greater promotion of nurse led clinics at initial assessments and clusters were reminded to consider clinics during team meetings and at MDT.

A second audit confirmed an increase in the use of nurse led clinic slots:

- 23/6/14 - 31/12/14 (6 month period) 51 clinic appointments booked ( $1^{\text {st }}$ audit)

- $1 / 6 / 15$ - 31/8/15 (3 month period) 101 clinic appointments booked ( $2^{\text {nd }}$ audit)

Failure to attend clinic appointments were relatively low at $11 \%$.

Changes in the service model have:

- provided patients and their carers with greater accessibility to the HNST

- increased responsiveness to needs

- given patients more choice about their care provision

- patients have expressed that clinics greatly reduced their fears of the hospice environment and services.

\section{P-198 YOU CAN LEAD A PATIENT TO A HOSPICE BUT YOU CAN'T MAKE THEM ENTER THE DOOR}

1,2Lynne Colbourne, 'Vanessa Gibson. 'St Richard's Hospice, Worcester, UK: ${ }^{2}$ Worcestershire Acute Hospitals NHS Trust

10.1136/bmjspcare-2016-001245.220

Patients diagnosed with metastatic breast cancer can experience years living with their cancer (Reed and Corner 2012). This group are often referred late in their cancer trajectory to palliative services. In an attempt to reverse this trend a Secondary Breast Cancer CNS (SBCCNS) post was funded by the local hospice as a three-year fixed term trial. The SBCCNS works as a member of the hospice and oncology team providing an in-reach key worker role to oncology clinics and wards.

The evaluation of the role is on-going. Initial review after six months of the role has identified the following benefits:

- Patients are providing positive feedback. Having a named key worker who can be available in primary and secondary care is seen as reassuring.

- Undertaking joint home review visits with the primary care CNS. Improve communication between the primary and secondary care teams.

- Holistic needs assessment and individualised care planning that encourages early referral to services including financial assessment, family support/counselling, dietetic input etc.

- Instances of admission avoidance to the acute Trust or directing admission to an appropriate setting e.g. hospice inpatient unit.

- Offering an alternative venue in day hospice for symptom control clinics and blood transfusion administration.
Patients with secondary breast cancer may spend years receiving anti-cancer treatment and trying to live their lives. This can result in reduced access to palliative services provided by hospice teams. Taking the service to the patient and introducing the potential benefits of palliative care services appears to be an initiative that patients accept.

\section{P-199 ABSTRACT WITHDRAWN}

\section{P-200 DEVELOPMENT OF A RAPID RESPONSE TEAM TO FACILITATE 24 HOUR END OF LIFE CARE FOR CHILDREN AND YOUNG PEOPLE IN THEIR PREFERRED PLACE OF CARE}

Lesley Fellows. Claire House Children's Hospice, Bebington, UK

10.1136/bmjspcare-2016-001245.221

Background Prior to the Merseyside End of Life (EOLC) pilot, palliative and end-of-life care for children and young people (CYP) across NHS Merseyside has been provided by the specialist palliative care team for advice and home assessments visits on a 24/7 basis and supported on an ad hoc basis by local Children's Community Nursing (CCN) teams and/or adult district nurses.

None of the existing CCN services across this footprint (6 Clinical Commissioning Groups - CCGs) were commissioned to provide the 'step up' support for EOLC at home, including the availably of 24/7 CCN support, which potentially limited realistic choice in place for EOLC for children and their families.

Unlike adult EOLC one of the major challenges of providing care for children is that diagnosing dying is difficult as children often have peaks and troughs in their condition and therefore, sustainable services that are responsive to provide a step up, step down approach are essential.

The North Mersey Paediatric QIPP programme identified specific gaps in service provision for EOL care and also reflected on the small numbers of children's deaths for each CCG. They noted that there were significant challenges for existing CCNT to maintain skills and expertise in this specialist field and a potential solution was a collaborative partnership approach with Claire House $(\mathrm{CH})$.

The proposed pilot was for a Hospice to Home Team who are skilled and experienced practitioners to provide a 24-hour rapid response service for EOLC across the Mersey footprint, working in partnership with existing CCN and SPC Teams.

The aim was to facilitate this for between 15 and 23 children, enabling them to have an equitable, responsive and realistic choice for EOLC at home. This development supported the increase in home based EOLC to meet the best in England.

In order to make a success of the pilot collaborative working was key. An operational stakeholder group was formed which had representation from hospice, SPCT and CCNT. Shared referral pathways, key communication messages, procedures and policies were jointly formulated and endorsed. This group met fortnightly initially whilst essential operational documents and governance issues were discussed then as the pilot became open to referrals this became monthly. Case discussion and reflection was paramount for group learning and for continuous service improvements.

A steering group provided a mechanism for feedback on the pilot's progress and monitored governance, risk and acted as a critical friend. The steering group ensured that preparations were 